\title{
Festina-Lente bound on Higgs vacuum structure and inflation
}

\author{
Sung Mook Lee, ${ }^{a}$ Dhong Yeon Cheong, ${ }^{a}$ Sang Chul Hyun, ${ }^{a}$ Seong Chan Park ${ }^{a, b, 1}$ \\ and Min-Seok Seo ${ }^{c, 1}$ \\ ${ }^{a}$ Department of Physics $\&$ IPAP $\&$ Lab for Dark Universe, Yonsei University, \\ Seoul 03722, Republic of Korea \\ ${ }^{b}$ Korea Institute for Advanced Study, \\ Seoul 02455, Republic of Korea \\ ${ }^{c}$ Department of Physics Education, Korea National University of Education, \\ Cheongju 28173, Republic of Korea \\ E-mail: sungmook.lee@yonsei.ac.kr, dhongyeon@yonsei.ac.kr, \\ bsg04103@yonsei.ac.kr, sc.park@yonsei.ac.kr, minseokseo57@gmail.com
}

ABstract: The recently suggested Festina-Lente (FL) bound provides a lower bound on the masses of $U(1)$ charged particles in terms of the positive vacuum energy. Since the charged particle masses in the Standard Model (SM) are generated by the Higgs mechanism, the FL bound provides a testbed of consistent Higgs potentials in the current dark energydominated universe as well as during inflation. We study the implications of the FL bound on the UV behavior of the Higgs potential for a miniscule vacuum energy, as in the current universe. We also present values of the Hubble parameter and the Higgs vacuum expectation value allowed by the FL bound during inflation, which implies that the Higgs cannot stay at the electroweak scale during this epoch.

Keywords: Beyond Standard Model, Cosmology of Theories beyond the SM, Effective Field Theories, Higgs Physics

ArXiv EPrint: 2111.04010

\footnotetext{
${ }^{1}$ Corresponding author.
} 


\section{Contents}

1 Introduction 1

2 Structure of Higgs vacua $\quad 3$

2.1 Higgs potential 3

2.2 Implications of FL bound 4

2.2.1 Case 1: single vacuum at $h=v_{\mathrm{EW}} \quad 5$

2.2.2 Case 2: two vacua at $h=v_{\mathrm{EW}}$ and $v_{\mathrm{UV}}>v_{\mathrm{EW}}$

2.2.3 Case $2^{\prime}$ : (nearly) degenerate vacua, $v_{\mathrm{EW}} \simeq v_{\mathrm{UV}}$

$\begin{array}{lll}2.2 .4 & \text { Case } 2^{\prime \prime} \text { : inflection point at } v_{\mathrm{UV}} & 7\end{array}$

$\begin{array}{lll}3 & \text { FL bound and inflation } & 7\end{array}$

$\begin{array}{llr}4 & \text { Conclusion } & 9\end{array}$

A Hawking-Moss and Coleman-de Luccia instanton near inflection point 10

\section{Introduction}

Can our understanding of the universe based on low energy dynamics be completed up to quantum gravity? This is one of main themes of the swampland program, which provides conjectured constraints on the low energy effective field theories (EFT) to be consistent with the UV completion of quantum gravity [1]. (For recent reviews, see refs. [2-4]. Also see refs. [5-7] for specific inflationary models.) While many conjectures are based on string compactification, some of them are motivated based on a more generic quantum gravity context, including black hole $(\mathrm{BH})$ physics. For instance, although string theory claims that de Sitter (dS) space is unstable [8] (see also refs. [9-11]), the universe may stay in quasi-dS for a sufficiently long enough time $[12,13]$. Then (quasi-)dS can be approximated as a stable background and the dS-BH solutions as well as their thermal behavior can be used to set bounds on the low energy parameters (see, for example, ref. [14] and also ref. [15] for a discussion concerning $\mathrm{dS}$ instability).

If a dS-BH is super-extremal, i.e., the BH horizon is not generated inside the cosmological horizon, the BH singularity is naked. This has been widely considered to be forbidden as claimed by the cosmic censorship hypothesis [16], motivated by the predictability from the initial data as well as the observational consistency so far. In order to avoid the naked singularity, we can impose that the charged Nariai BH in which the $\mathrm{BH}$ horizon coincides with the cosmological horizon must discharge without losing sub-extremality. ${ }^{1}$ From this,

\footnotetext{
${ }^{1}$ On the other hand, imposing sub-extremality for $\mathrm{BH}$ much smaller than dS horizon size provides Weak Gravity Conjecture [17].
} 
one obtains the Festina Lente (FL) bound $[18,19]$ : the mass $m$ for every state of charge $q$ under $\mathrm{U}(1)$ gauge invariance with coupling $g$ satisfies

$$
\frac{m^{4}}{8 \pi \alpha q^{2}} \geq V \geq 0
$$

where $\alpha \equiv \frac{g^{2}}{4 \pi}$ and $V=3 M_{P}^{2} H^{2}(H$ : Hubble parameter) is the energy density governing Hubble expansion. One direct implication is that the massless charged state is forbidden for nonzero $V$. This bound also forbids unwanted charged black hole remnants.

In the Standard Model (SM) of particle physics, U(1) gauge invariance corresponds to electromagnetism, which is generated through the spontaneous breaking of electroweak gauge invariance via the Higgs mechanism. Then every $U(1)$ charged particle mass is proportional to the Higgs vacuum expectation value (vev). Moreover, the cosmic energy density of the current universe is dominated by dark energy $V=\Lambda_{\mathrm{DE}} \sim 10^{-120} M_{P}^{4}$. These two are consistent with the FL bound as the masses of the charged particles in the SM are all many orders larger than the Hubble scale of the current universe $H_{0} \sim \mathcal{O}\left(10^{-60} M_{P}\right)$ [19].

On the other hand, under the desert scenario which assumes the absence of any new physics up to the grand unification or the Planck scale, the self quartic coupling of the Higgs may vanish at UV and such near criticality of the Higgs potential raises the Higgs vacuum stability issue [20-23]. Also, depending sensitively on low energy SM parameters, the Higgs potential may develop another (meta-)stable vacuum at UV, at which the masses of the $\mathrm{U}(1)$ charged particles are enhanced.

In this case, the FL bound is satisfied only if the vacuum energy density contribution from the Higgs potential is tuned such that even if it dominates the dark energy, its value is still tiny. The UV Higgs vev is fixed in accordance with this requirement. In this work, we investigate such constraints more concretely by considering possible UV behaviors of the Higgs potential [22-24].

Moreover, it is plausible to postulate the inflationary epoch at the early stage of the universe, where the universe was in a quasi-dS phase. This not only resolves the horizon and flatness problems [25-27], but also explains how quantum fluctuations become the seed for large scale structures $[28,29]$. Intriguingly, whereas we typically assume the reheating temperature $T_{\text {reh }}$ after the inflation to be larger than the Big Bang nucleosynthesis (BBN) scale of $\mathcal{O}(10 \mathrm{MeV})$, the vacuum energy in this case is too large to satisfy the FL bound if the electron mass remains the same or the Higgs vev is given by the electroweak scale. This implies that during inflation, the Higgs potential should be stabilized at the UV vev, so that the electron mass (the lightest $\mathrm{U}(1)$ charged particle in SM) becomes heavy to satisfy the FL bound. Regarding this, we discuss the restriction of the FL bound on the UV Higgs vev and the vacuum energy density during inflation.

The paper is organized as follows. In section 2, we discuss the applicability of the FL bound for various possible UV behaviors of the Higgs and obtain the FL bound constraints on the structure of the Higgs potential. In section 3, we present the FL bound constraints on the Higgs vev and the vacuum energy during the inflation, together with relevant cosmological observables. We conclude in section 4 . 


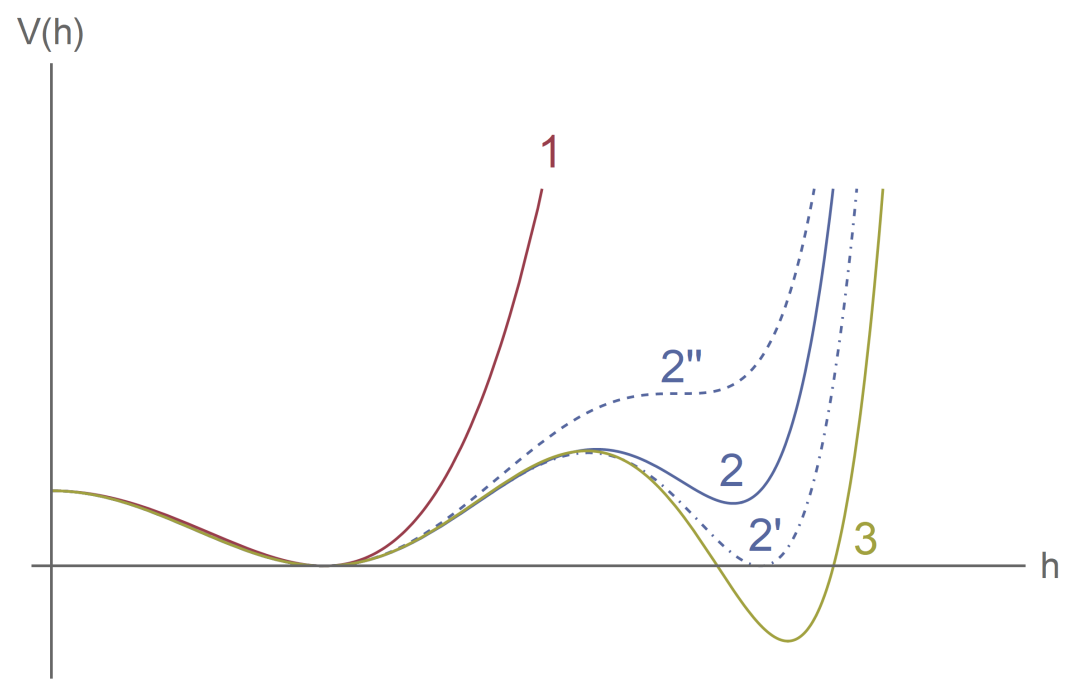

Figure 1. Schematic shape of the Higgs potential.

\section{Structure of Higgs vacua}

The shape of the Higgs potential at UV $\left(h \gg v_{\mathrm{EW}}\right)$ that is consistent with low energy SM parameter values is not precisely determined, yet. The allowed shapes are schematically depicted in figure 1. Even in the absence of new physics, the renormalization group (RG) running of the Higgs self quartic coupling $\lambda(\mu)$ depends sensitively on the SM parameters at electroweak (EW) scale, including $\mathcal{O}(1)$ top Yukawa coupling, i.e., the top quark mass. ${ }^{2}$ In addition, non-renormalizable operators that are irrelevant at the EW scale may become important at the UV scale as well. They allow various possible UV behaviors of the Higgs potential $[21,30]$.

Meanwhile, the FL bound comes from a gravitational argument, thus it is reasonable to assume that the FL bound can be applied to cases where the Higgs dominates the vacuum energy and the Higgs remains at some UV value for a sufficient amount of time. In this section, we discuss the applicability of the FL bound in these cases for each possible UV structure of the Higgs potential.

\subsection{Higgs potential}

Imposing $\mathbb{Z}_{2}$ symmetry, the UV Higgs potential we consider includes dark energy (DE), and higher order terms suppressed by cut-off scale $\Lambda$ as

$$
V_{\mathrm{eff}}(h)=\Lambda_{\mathrm{DE}}+\frac{\lambda(h)}{4} h^{4}+\frac{c_{6}}{\Lambda^{2}} h^{6}+\frac{c_{8}}{\Lambda^{4}} h^{8}+\cdots .
$$

The constant DE $\Lambda_{\mathrm{DE}} \sim 10^{-120} M_{P}^{4}$ will be neglected in the succeeding discussion. The effective potential can be comprehensively written by defining the effective quartic coupling as

$$
\lambda_{\text {eff }}(h) \equiv \frac{4 V_{\text {eff }}}{h^{4}} .
$$

\footnotetext{
${ }^{2}$ In our definition, $\lambda$ also includes the contribution from the correction to the 1PI effective action.
} 
Regarding the Higgs quartic coupling $\lambda(h)$ in eq. (2.1), we take the renormalization scale $\mu$ to be $h$ following refs. [21,31,32]. In the vicinity of some specific value $h=h_{*}$, which we usually take $h_{*}=h_{0}$ at which $\lambda\left(h_{0}\right)=0, \lambda(h)$ is expanded as

$$
\lambda(h)=\lambda\left(h_{*}\right)-\frac{b_{1}}{(4 \pi)^{2}} \ln \frac{h}{h_{*}}+\frac{b_{2}}{(4 \pi)^{4}} \ln ^{2} \frac{h}{h_{*}}+\cdots .
$$

If we consider the pure SM sector, $b_{1}=-(4 \pi)^{2} \beta_{\lambda}\left(h_{*}\right) \simeq 1 / 4$ where $\beta_{\lambda} \equiv d \lambda / d(\ln \mu)$ works quite well for our purposes. In the non-renormalizable terms $c_{2 n}(n \geq 3)$ are unknown $\mathcal{O}(1)$ coefficients. These higher order terms are also required to impose the stability of the Higgs potential when $\lambda(h)$ runs to negative values at high scales. In this work, to be explicit, we keep up to the dimension six operator setting $c_{6}=1$ and, $c_{8}=c_{10}=\cdots=0$ letting the cutoff scale $\Lambda$ a free parameter. ${ }^{3}$

Here, we schematically categorize the shapes of $V_{\text {eff }}$ in three distinctive cases:

- Case 1: $V_{\text {eff }}$ has a unique $\mathrm{EW}$ vacuum at $v_{\mathrm{EW}}$, hence the potential is monotonically increasing beyond $v_{\mathrm{EW}}$. As we will show, the FL bound cannot be applied for $h>v_{\mathrm{EW}}$ unless the potential is close to Case $2^{\prime \prime}$.

- Case 2: $V_{\text {eff }}$ is positive for all $h$ and has another local minimum at UV, $v_{\mathrm{UV}}$. There are two interesting limits for this case.

- Case 2': $V_{\text {eff }}$ possesses a (almost) degenerate Higgs vacua, i.e.,

$$
V_{\mathrm{eff}}\left(v_{\mathrm{UV}}\right) \simeq 0, \quad V_{\mathrm{eff}}^{\prime}\left(v_{\mathrm{UV}}\right)=0
$$

and the same relations hold for $v_{\mathrm{EW}}$.

- Case $2^{\prime \prime}: V_{\text {eff }}$ has an inflection point at which

$$
V_{\text {eff }}^{\prime}\left(v_{\text {inf }}\right)=0, \quad V^{\prime \prime}\left(v_{\text {inf }}\right)=0 .
$$

- Case 3: $V_{\text {eff }}$ has a UV local minimum $v_{\mathrm{UV}}$, but becomes negative around $v_{\mathrm{UV}}$. In this case, the FL bound is not applicable because the universe would be AdS rather than dS unless another source of vacuum energy is added. We will visit this case in section 3 .

Since we restrict our discussion to $\Lambda_{\mathrm{DE}} \simeq 0$ in this section, we consider the FL implications on Case 1 and Case 2.

\subsection{Implications of FL bound}

For the FL bound to be applied, by construction, the background of the universe should be close to dS for an extended period of time compared to the BH lifetime. ${ }^{4}$ This condition

\footnotetext{
${ }^{3}$ When $c_{2 n}$ with $n \geq 4$ are neglected, $c_{6}$ must be positive for the stability of the Higgs potential.

${ }^{4}$ More precisely, the background geometry after the charged $\mathrm{BH}$ production is required to be deformed close to that of the Nariai $\mathrm{BH}, \mathrm{dS}_{2} \times \mathrm{S}^{2}[18,19]$, which undoubtedly includes the nearly constant cosmological horizon case.
} 
can be comprehensively written in terms of the potential slow-roll parameter defined by

$$
\epsilon_{V} \equiv \frac{M_{P}^{2}}{2}\left(\frac{V_{\mathrm{eff}}^{\prime}(h)}{V_{\mathrm{eff}}(h)}\right)^{2}=\frac{8 M_{P}^{2}}{h^{2}}\left(1+\frac{\beta_{\lambda, \mathrm{eff}}}{4 \lambda_{\mathrm{eff}}}\right)^{2}
$$

where $\beta_{\lambda, \text { eff }} \equiv d \lambda_{\text {eff }} / d(\ln \mu)$. With $g V^{1 / 2}$ being the electric field for the Nariai BH, we should have

$$
\epsilon_{V} \ll e^{-\frac{m^{2}}{g q \sqrt{V}}}<1
$$

\subsubsection{Case 1: single vacuum at $h=v_{\mathrm{EW}}$}

In Case 1, the Higgs potential has one local minimum at $v_{\mathrm{EW}}$, then monotonically increases. The EW vacuum is consistent with the FL bound provided the dark energy is as small as $\Lambda_{\mathrm{DE}} \lesssim m_{e}^{4} /(8 \pi \alpha) \sim 10^{-88} M_{P}^{4}$ since the electron is the lightest $\mathrm{U}(1)$ charged particle in the SM. This bound is consistent with the current universe since $\Lambda_{\mathrm{DE}} \sim 10^{-120} M_{P}^{4}$ as measured from Planck [33] satisfies the bound, which is noticed earlier in ref. [19]. The validity during inflation will be discussed in detail in section 3 .

\subsubsection{Case 2: two vacua at $h=v_{\mathrm{EW}}$ and $v_{\mathrm{UV}}>v_{\mathrm{EW}}$}

We now turn our focus to Cases 2, $2^{\prime}$, and $2^{\prime \prime}$, where the Higgs potential has a (meta-)stable UV vacuum $v_{\mathrm{UV}}$ satisfying $V^{\prime}\left(v_{\mathrm{UV}}\right)=0$ (hence $\epsilon_{V}=0$ ) and $V\left(v_{\mathrm{UV}}\right) \geq 0$. Imposing the FL bound on the UV Higgs vacuum we obtain the upper bound on the effective quartic coupling $\lambda_{\text {eff }}\left(v_{\mathrm{UV}}\right)>0$ as

$$
\begin{aligned}
\min _{i \in \mathrm{SM}} \frac{m_{i}^{4}}{8 \pi \alpha_{i}} & =\frac{y_{e}^{4} v_{\mathrm{UV}}^{4} / 4}{8 \pi \alpha_{\mathrm{EM}}} \geq \frac{\lambda_{\mathrm{eff}}\left(v_{\mathrm{UV}}\right)}{4} v_{\mathrm{UV}}^{4} \\
\Rightarrow \quad \lambda_{\mathrm{eff}}\left(v_{\mathrm{UV}}\right) & \leq \frac{y_{e}^{4}}{8 \pi \alpha_{\mathrm{EM}}} \simeq \mathcal{O}\left(10^{-22}\right) .
\end{aligned}
$$

Here the minimum of $m_{i}^{4} / \alpha_{i}$ is given by the SM electron which obtains its mass through the Higgs mechanism,

$$
m_{e}(h)=\frac{y_{e}(h)}{\sqrt{2}} h .
$$

We note that there are several theoretical arguments justifying this seemingly fine-tuned small $\lambda_{\mathrm{eff}}\left(v_{\mathrm{UV}}\right)$, which is out of the scope of this work (see, for example, ref. [34]).

\subsubsection{Case $2^{\prime}$ : (nearly) degenerate vacua, $v_{\mathrm{EW}} \simeq v_{\mathrm{UV}}$}

The degenerate case ( $V_{\text {eff }} \simeq 0$ at $h_{1}=v_{\mathrm{EW}}$ and $\left.h_{2}=v_{\mathrm{UV}}\right)$ satisfies the FL bound in a trivial manner. When $V_{\text {eff }}\left(v_{\mathrm{UV}}\right)$ is slightly uplifted, it does not violate the FL bound provided $V$ is smaller then $10^{-22} v_{\mathrm{UV}}^{4}$, where we expect that $v_{\mathrm{UV}}$ in this case can be approximated by the values in the degenerate case. Given

$$
\lambda(h)=\lambda_{*}-\frac{b_{1}}{(4 \pi)^{2}} \ln \left(\frac{h}{h_{*}}\right), \quad V_{\mathrm{eff}}(h)=\frac{\lambda(h)}{4} h^{4}+\frac{1}{\Lambda^{2}} h^{6},
$$




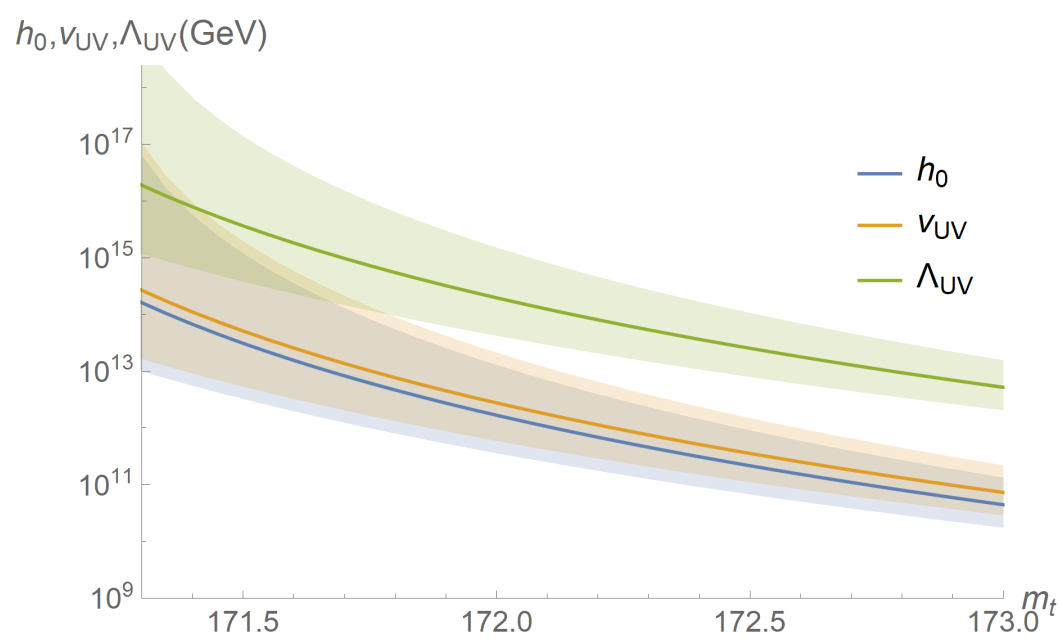

Figure 2. Scales for $h_{0}, v_{\mathrm{UV}}, \Lambda_{\mathrm{UV}}$ depending on the top quark mass. Large additional uncertainty comes from strong coupling constant, which is depicted as shaded region at $1 \sigma$ level with $\alpha_{s}=0.1179 \pm 0.0010[35]$.

we estimate the values of $v_{\mathrm{UV}}$ and the cut-off scale $\Lambda$ from the conditions $V_{\text {eff }}\left(v_{\mathrm{UV}}\right)=0=$ $V_{\text {eff }}^{\prime}\left(v_{\mathrm{UV}}\right)$ as

$$
\begin{aligned}
v_{\mathrm{UV}} & =h_{*} \exp \left(\frac{16 \pi^{2} \lambda_{*}}{b_{1}}+\frac{1}{2}\right) \\
\Lambda_{\mathrm{UV}} & =\frac{8 \sqrt{2} \pi}{\sqrt{b_{1}}} h_{*} \exp \left(\frac{16 \pi^{2} \lambda_{*}}{b}+\frac{1}{2}\right)=\frac{8 \sqrt{2} \pi}{\sqrt{b_{1}}} v_{\mathrm{UV}} .
\end{aligned}
$$

Note that both $v_{\mathrm{UV}}$ and $\Lambda_{\mathrm{UV}}$ depend on the combination $h_{*} \exp \left(16 \pi^{2} \lambda_{*} / b_{1}\right)$ which is nothing more than $h \exp \left(16 \pi^{2} \lambda(h) / b_{1}\right)$. Thus they are independent on the choice of the fiducial value $\lambda_{*}$, or equivalently $h_{*}$ for the RG running, as expected. Our estimation fixes the value of $\lambda\left(v_{\mathrm{UV}}\right)$, which is physically meaningful, to be

$$
\lambda\left(v_{\mathrm{UV}}\right)=-\frac{b_{1}}{32 \pi^{2}} .
$$

Since $\lambda\left(v_{\mathrm{UV}}\right)$ is negative, it is cancelled with $\left(v_{\mathrm{UV}} / \Lambda_{\mathrm{UV}}\right)^{2}$ to give $V_{\mathrm{eff}}\left(v_{\mathrm{UV}}\right)=0$ or $\lambda_{\mathrm{eff}}\left(v_{\mathrm{UV}}\right)=$ $\lambda\left(v_{\mathrm{UV}}\right)+\left(v_{\mathrm{UV}} / \Lambda_{\mathrm{UV}}\right)^{2}=0$.

One convenient choice of $h_{*}$ is the value of the Higgs $h_{0}$ giving $\lambda\left(h_{0}\right)=0$, in terms of which we obtain

$$
v_{\mathrm{UV}}=\sqrt{e} h_{0}, \quad \Lambda_{\mathrm{UV}}=\frac{8 \sqrt{2 e} \pi}{\sqrt{b_{1}}} h_{0} .
$$

While the RG running of the SM parameters gives $h_{0} \gtrsim 10^{10} \mathrm{GeV}$, the explicit value of $h_{0}$ is sensitive to the top quark mass. Explicit values of $\left(h_{0}, v_{\mathrm{UV}}, \Lambda_{\mathrm{UV}}\right)$ depending on the EW top quark mass with uncertainty from strong coupling constant are shown in figure 2 . 


\subsubsection{Case $2^{\prime \prime}$ : inflection point at $v_{\mathrm{UV}}$}

We now consider the Higgs potential shape close to Case $2^{\prime \prime}$ in which the inflection point exists. By setting $\lambda_{*}=\lambda_{0}=0$ we obtain from the conditions $V_{\text {eff }}^{\prime}\left(v_{\text {inf }}\right)=0$ and $V_{\text {eff }}^{\prime \prime}\left(v_{\text {inf }}\right)=0$ that $v_{\text {inf }}$ and the cutoff scale $\Lambda_{\text {inf }}$ are given by

$$
v_{\mathrm{inf}}=e^{1 / 4} h_{0}, \quad \Lambda_{\mathrm{inf}}=\frac{8 \sqrt{3} \pi e^{1 / 4}}{\sqrt{b_{1}}} h_{0} .
$$

Then the value of the potential height at $v_{\text {inf }}$ is,

$$
V_{\text {eff }}\left(v_{\text {inf }}\right)=\frac{b_{1} e}{768 \pi^{2}} h_{0}^{4} .
$$

Since $\lambda_{\text {eff }}\left(v_{\text {inf }}\right)=\frac{b_{1}}{192 \pi^{2}} \simeq \mathcal{O}\left(10^{-4}\right) \gg 10^{-22}$, we conclude that the vacuum energy at the inflection point is too large to satisfy the FL bound. We also note that the inflection point is not consistent with the dS swampland conjecture, the refined version of which requires $\eta_{V}\left(v_{\text {inf }}\right)<-\mathcal{O}(1)$ even if $\epsilon_{V}\left(v_{\text {inf }}\right) \ll 1[9-11]$.

We also note that the stability of the vacuum in Case 2 discussed in this section is purely classical. However, especially for setups near the inflection point, tunneling via a Coleman-de Luccia or Hawking-Moss instanton may induce the UV vacuum to decay faster than the charged BHs, such that the FL bound may not be applicable. This case includes the inflection point itself. Therefore, even with a large $\lambda_{\text {eff }}$, we cannot rule out Case $2^{\prime \prime}$ by the FL bound. See appendix A for details.

\section{FL bound and inflation}

In this section, we consider additional scalar fields $\varphi_{i}, i=1,2, \cdots$, which, in addition to the Higgs field, contribute to the Hubble expansion especially during inflationary epoch:

$$
\sum_{i} U_{i}\left(\varphi_{i}\right)+V(h)=3 M_{P}^{2} H_{I}^{2}
$$

where $H_{I}$ is the (nearly constant) Hubble parameter. Then the FL bound is found taking the electron mass given by the Higgs mechanism with vacuum expectation value $h$, as

$$
\frac{y_{e}^{4} h^{4} / 4}{8 \pi \alpha_{\mathrm{EM}}} \geq 3 M_{P}^{2} H_{I}^{2}
$$

or

$$
h \geq\left(\frac{96 \pi \alpha_{\mathrm{EM}}}{y_{e}^{4}}\right)^{1 / 4} \sqrt{M_{P} H_{I}} .
$$

The region excluded by the FL bound is colored in blue in figure 3 . For $h<M_{P}$, using $y_{e}\left(M_{P}\right) \simeq 2.7 \times 10^{-6}$ and $\alpha_{\mathrm{EM}}\left(M_{P}\right) \simeq 9.5 \times 10^{-3}$, we obtain a strong bound on the Hubble parameter during inflation

$$
H_{I} \lesssim 10^{7} \mathrm{GeV}
$$




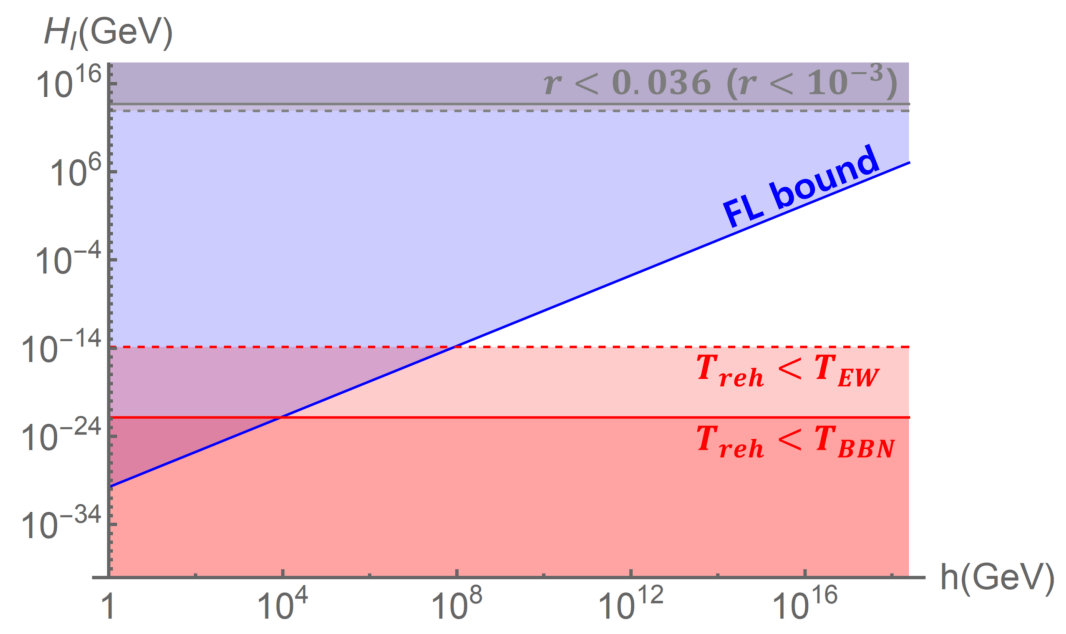

Figure 3. Bound on inflation scale from FL bound depending on the location of Higgs vev. Only white colored region is allowed.

which is much more stringent than the conventionally known bound $H_{I}<10^{13-14} \mathrm{GeV}$ from the recent Planck+BICEP/Keck 2018 observations [36].

If the Higgs stays at the EW vacuum during the inflation $\left(h=v_{\mathrm{EW}}\right)$, the FL bound is satisfied provided $\sum_{i} U_{i}\left(\varphi_{i}\right)+V(h) \lesssim 10^{-88} M_{P}^{4}$, or equivalently, $H_{I} \lesssim 10^{-44} M_{P} \simeq$ $10^{-26} \mathrm{GeV}$. However, this is in tension with the typical inflation scenario, which requires the reheating temperature $T_{\text {reh }}$ to be larger than the BBN scale. To see this, recall that the instantaneous reheating temperature is determined under the situation where the inflation energy density is immediately converted into radiation, $\rho_{\text {inf }} \simeq \rho_{\text {rad }}$, thus

$$
3 M_{P}^{2} H_{I}^{2} \simeq \frac{\pi^{2}}{30} g_{\mathrm{reh}} T_{\mathrm{reh}, \mathrm{inst}}^{4}
$$

where $g_{\text {reh }}$ is the effective degree of freedom during reheating. In general, the actual reheating temperature is upper bounded as $T_{\text {reh }} \leq T_{\text {reh,inst }}$. The FL bound implies a lower bound on the vacuum expectation value during inflation in terms of the reheating temperature

$$
h \geq\left(\frac{16 \pi^{3} g_{\mathrm{reh}} \alpha_{\mathrm{EM}}}{15 y_{e}^{4}}\right)^{1 / 4} T_{\mathrm{reh}} \simeq 10^{6} \cdot T_{\mathrm{reh}} .
$$

Requesting the reheating temperature to be larger than the BBN scale $T_{\text {reh }} \geq T_{\mathrm{BBN}} \sim 10 \mathrm{MeV}$, we obtain

$$
h \gtrsim 10^{4} \mathrm{GeV}, \quad \text { (during inflation) }
$$

which is much larger than $v_{\mathrm{EW}} \simeq 246 \mathrm{GeV}$. Therefore, under reasonably acceptable conditions, the Higgs does not seem to stay at the electroweak vacuum during inflation. ${ }^{5}$

\footnotetext{
${ }^{5}$ If the Hubble scale is larger than the Higgs vev, the spontaneously broken EW gauge invariance is restored. The FL bound imposes that the Higgs vev to be larger than $H_{I}$ during the inflation, which excludes this possibility.
} 
The region ruled out by this requirement is colored in red in figure 3 . We also depicted the case with the more stringent requirement $T_{\text {reh }}>T_{\mathrm{EW}} \sim 100 \mathrm{GeV}$ assumed.

After inflation, EW vacuum may be restored during the early thermal history of our universe, without any violation of the FL bound due to the departure from the (quasi-) dS phase.

We also comment on the supersymmetric extension of the SM and its breaking mechanism. If we consider the gravity mediation [37] of the SUSY breaking to the SM sector, the Higgs soft mass is typically in the Hubble scale, $H_{I}$. While the Higgs quartic coupling in the minimal supersymmetric extension is given by the gauge coupling, it can vanish when the Higgs is on the D-flat direction. Then the quartic term is dominated by $\lambda \sim H_{I}^{2} / \Lambda^{2}$ such that the Higgs potential during inflation, schematically written in the form of

$$
V(h)=-\frac{c_{2}^{2}}{2} H_{I}^{2} h^{2}+\frac{1}{4} \frac{H_{I}^{2}}{\Lambda^{2}} h^{4}, \quad c_{2}>0
$$

is stabilized at $v_{\mathrm{UV}}=c_{2} \Lambda$, which can be larger than eq. (3.3) thus satisfies the FL bound. For gauge mediation [38], on the other hand, the soft mass $H_{I} M_{P} / M$ can be enhanced by the sub-Planckian messenger scale $M$, such that the Higgs vev satisfies eq. (3.3) for $M<\left(H_{I} M_{P}\right)^{1 / 2}$ even with a $\mathcal{O}(1)$ Higgs quartic coupling.

The bounds on $H_{I}$ and $h$ we obtained above is consequentially encoded in bounds of inflationary observables, which enables us to test the FL bound. The scalar(tensor) power spectrum of the quantum fluctuation during inflation is usually parametrized by the scalar(tensor) amplitude $A_{s}\left(A_{t}\right)$ and spectral indices $n_{s}\left(n_{t}\right)$ with a pivot scale $k_{*}$ as

$$
\mathcal{P}_{\mathcal{S}}(k)=A_{s}\left(\frac{k}{k_{*}}\right)^{n_{s}-1}, \quad \mathcal{P}_{\mathcal{T}}(k)=A_{t}\left(\frac{k}{k_{*}}\right)^{n_{t}}
$$

and the tensor-to-scalar ratio is defined by $r \equiv A_{t} / A_{s}$. The Planck+BICEP/Keck 2018 result provides $A_{s} \simeq 2.1 \times 10^{-9}$ and a bound on $r<0.036$ with $k_{*}=0.05 \mathrm{Mpc}^{-1}[33,36]$.

For the single field slow-roll inflation, the bound on the Hubble scale is directly converted to the bound on the tensor-to-scalar ratio using the relation

$$
\frac{H_{I}^{2}}{M_{P}^{2}} \simeq \frac{\pi^{2}}{2} A_{s} r
$$

as

$$
r \lesssim 3 \times 10^{-15}\left(\frac{10^{-2}}{\alpha_{\mathrm{EM}}}\right)\left(\frac{2 \cdot 10^{-9}}{A_{s}}\right)\left(\frac{y_{e}}{3 \cdot 10^{-6}}\right)^{4}\left(\frac{h}{M_{P}}\right)^{4}
$$

which is many orders smaller than the observability of current/proposed experiments [36, 39-41].

\section{Conclusion}

In this work, we analyze the implications of the recently proposed FL bound on the Higgs vacuum structure and the inflationary cosmology. Among the possible structures the UV Higgs potential may have (as shown in figure 1), the FL bound can be applied to Case 2, in 
which another positive local minimum appears at UV. The FL bound restricts $V_{\text {eff }}\left(v_{\mathrm{UV}}\right)$ to be almost vanishing such that the UV vacuum is nearly degenerate with the EW vacuum. The sizes of $v_{\mathrm{UV}}$ and $\Lambda_{\mathrm{UV}}$ are also constrained, the exact values of which sensitively depend on the top quark mass, as well as the strong coupling constant.

Meanwhile, unlike the current universe, the EW vacuum is not compatible with the vacuum energy when the universe was in the inflationary phase provided the reheating temperature is larger than the BBN scale. To satisfy the FL bound, the Higgs should have a vacuum at UV scale, satisfying $h \gtrsim 10^{4} \mathrm{GeV}$ and the Hubble scale $H_{I}$ is required to be less than the order of $10^{7} \mathrm{GeV}$, implying minuscule tensor-to-scalar ratio $r \lesssim 3 \times 10^{-15}$.

\section{Acknowledgments}

We are grateful to Misao Sasaki and Chang Sub Shin for discussions and valuable comments. This work was supported by National Research Foundation grants funded by the Korean government (MSIT) (NRF-2019R1A2C1089334), (NRF-2021R1A4A2001897) (SCP), (MOE) (NRF-2020R1A6A3A13076216) (SML), and (NRF-2021R1A4A5031460) (MS). The work of SML is supported by the Hyundai Motor Chung Mong-Koo Foundation Scholarship.

\section{A Hawking-Moss and Coleman-de Luccia instanton near inflection point}

In this appendix, we quantitatively estimate the UV vacuum transition rate to EW vacuum near the inflection point and find the conditions for the Higgs potential to be stable, even quantum mechanically.

For a given decay rate, we denote $\Gamma \simeq A e^{-B}$. From $\mathrm{FL}$, the Nariai BH decay rate is $[18,19]$

$$
\Gamma_{\mathrm{BH}} \sim \exp \left(-\frac{m^{2}}{g q \sqrt{V}}\right) \sim \exp \left(-\frac{m^{2}}{g q M_{P} H}\right) .
$$

To ensure the FL bound, stability of UV vacuum should be guaranteed, i.e. $\Gamma_{\mathrm{BH}} \gg \Gamma_{\mathrm{dS}}$, hence $B_{\mathrm{BH}} \ll B_{\mathrm{dS}}$.

For the inflection point, we have

$$
v_{\text {inf }}=e^{1 / 4} h_{0}, \quad \Lambda_{\text {inf }}=\frac{8 \sqrt{3} \pi e^{1 / 4}}{\sqrt{b_{1}}} h_{0}=\frac{8 \sqrt{3} \pi}{\sqrt{b_{1}}} v_{\text {inf }},
$$

with

$$
V_{\text {inf }}=\frac{b_{1}}{768 \pi^{2}} v_{\text {inf }}^{4}, \quad H=\frac{\sqrt{b_{1}} v_{\text {inf }}^{2}}{48 \pi M_{P}}
$$

and

$$
\lambda_{\mathrm{eff}}=\frac{4 V_{\mathrm{inf}}}{v_{\mathrm{inf}}^{4}}=\frac{b_{1}}{192 \pi^{2}} \sim 10^{-4}
$$

Small deviation of $\Lambda_{\text {inf }}$ as

$$
\Lambda=\Lambda_{\text {inf }}\left(1+\delta_{\Lambda}\right)
$$


induces deviations for the field values at local maximum and local minimum

$$
h_{\max } \simeq v_{\text {inf }}\left(1-\delta_{v}\right), \quad h_{\min } \simeq v_{\text {inf }}\left(1+\delta_{v}\right)
$$

and $\Delta h \equiv h_{\max }-h_{\min } \simeq 2 v_{\text {inf }} \delta_{v}$ with $\delta_{v}=\sqrt{\delta_{\Lambda}}$ at the lowest order. Putting this,

$$
\delta_{\lambda} \equiv \frac{\delta \lambda_{\text {eff }}}{\lambda_{\text {eff }}}=-4 \delta_{v}
$$

and

$$
\Delta V \simeq \frac{b_{1} v_{\mathrm{inf}}^{4}}{12 \pi^{2}} \delta_{v}^{3}
$$

Near the inflection point, the relevant vacuum transition process is due to Coleman-de Luccia (CdL) and/or Hawking-Moss (HM) instanton respectively with

$$
B_{\mathrm{CdL}} \simeq \frac{2 \pi^{2} \sqrt{2 \Delta V}(2 \Delta h)}{H^{3}}, \quad \quad B_{\mathrm{HM}} \simeq \frac{8 \pi^{2} \Delta V}{3 H^{4}}
$$

$[22,42]$. Since the ratio of the two quantities is given as

$$
\frac{B_{\mathrm{CdL}}}{B_{\mathrm{HM}}} \simeq \frac{3 H \Delta h}{\sqrt{2 \Delta V}} \simeq \frac{\sqrt{3}}{4 \sqrt{2} \sqrt{\delta_{v}}}\left(\frac{v_{\mathrm{inf}}}{M_{P}}\right)
$$

the decay of false vacuum is dominated by $\mathrm{CdL}$ or $\mathrm{HM}$ instanton depending on the numerical value of $\delta_{v}$. When $\delta_{v} \simeq \delta_{v}^{\text {eq }}=\frac{3}{32}\left(\frac{v_{\text {inf }}}{M_{P}}\right)^{2}, B_{\mathrm{HM}} \simeq B_{\mathrm{CdL}}$ thus both are equally important. ${ }^{6}$

However, this result does not contain stochastic effects which are usually caught by Fokker-Planck (FP) approach. If $H^{2} \lesssim \sqrt{\Delta V}$ corresponding to

$$
\delta_{v} \gtrsim \frac{1}{48}\left(\frac{b_{1}}{4 \pi^{2}}\right)^{1 / 3}\left(\frac{v_{\text {inf }}}{M_{P}}\right)^{4 / 3} \simeq 4 \times 10^{-3}\left(\frac{v_{\text {inf }}}{M_{P}}\right)^{4 / 3},
$$

only considering a single CdL or HM would be enough [22]. On the other hand, if $H^{2} \gtrsim \sqrt{\Delta V}$, one has to consider FP process more carefully to determined the stability of the UV vacuum. For our purpose to see the smallness of the correction, it suffices to only consider the single CdL or HM instanton process.

For this case, to guarantee the stability of UV vacuum, we should have

$$
B_{\mathrm{BH}}=\frac{m^{2}}{g q M_{P} H} \ll\left\{\begin{aligned}
B_{\mathrm{CdL}} & \simeq \frac{2^{14} 3^{2} \sqrt{6} \pi^{4}}{b_{1}}\left(\frac{M_{P}}{v_{\text {inf }}}\right)^{3} \delta_{v}^{5 / 2}, \\
B_{\mathrm{HM}} & \simeq \frac{2^{17} 3^{2} \pi^{4}}{b_{1}}\left(\frac{M_{P}}{v_{\text {inf }}}\right)^{4} \delta_{v}^{3}
\end{aligned}\right.
$$

${ }^{6}$ We also note that the HM solution contributes to the vacuum decay provided $H>H_{\text {crit }}$ with

$$
H_{\text {crit }} \equiv \sqrt{-\frac{V^{\prime \prime}\left(v_{\max }\right)}{4}-\frac{\Delta V}{3 M_{p}^{2}}} \simeq \frac{\sqrt{b_{1}} v_{\text {inf }}}{4 \sqrt{2} \pi} \sqrt{\delta_{v}}, \quad \delta_{v}<\frac{1}{72}\left(\frac{v_{\text {inf }}}{M_{P}}\right)^{2} \equiv \delta_{v}^{\text {crit }}
$$

[43, 44], while for $H<H_{\text {crit }}$ the CdL solution contributes exclusively. Our estimation in eq. (A.9) is consistent to the fact that CdL and HM solutions usually coincide at the limit of $H \rightarrow H_{\text {crit }}$ as $\delta_{v}^{\text {eq }} \simeq \delta_{v}^{\text {crit }}$ [45]. 
implying

$$
\delta_{v} \gg \begin{cases}\left(\frac{b_{1} y_{e}^{4} v_{\mathrm{inf}}^{6}}{2^{23} 3^{3} \pi^{7} \alpha_{\mathrm{EM}} M_{P}^{6}}\right)^{1 / 5} \simeq 3 \times 10^{-7}\left(\frac{v_{\mathrm{inf}}}{M_{P}}\right)^{6 / 5} \quad \text { (CdL dominant) } \\ \left(\frac{b_{1} y_{e}^{4} v_{\mathrm{inf}}^{8}}{2^{28} 3^{2} \pi^{7} \alpha_{\mathrm{EM}} M_{P}^{8}}\right)^{1 / 6} \simeq 2 \times 10^{-6}\left(\frac{v_{\mathrm{inf}}}{M_{P}}\right)^{4 / 3} \quad \text { (HM dominant) }\end{cases}
$$

As expected, $\mathrm{dS}$ vacuum transition rates are suppressed as $\delta_{v}$ increase. Therefore, under the consideration of CdL and HM instanton, the UV vacuum is stable as long as eq. (A.11) holds. This already shows that our results numerically do not change much even when we consider the quantum effects.

Open Access. This article is distributed under the terms of the Creative Commons Attribution License (CC-BY 4.0), which permits any use, distribution and reproduction in any medium, provided the original author(s) and source are credited.

\section{References}

[1] C. Vafa, The String landscape and the swampland, hep-th/0509212 [INSPIRE].

[2] T.D. Brennan, F. Carta and C. Vafa, The String Landscape, the Swampland, and the Missing Corner, PoS TASI2017 (2017) 015 [arXiv:1711.00864] [INSPIRE].

[3] E. Palti, The Swampland: Introduction and Review, Fortsch. Phys. 67 (2019) 1900037 [arXiv: 1903.06239] [INSPIRE].

[4] M. Graña and A. Herráez, The Swampland Conjectures: A Bridge from Quantum Gravity to Particle Physics, Universe 7 (2021) 273 [arXiv:2107.00087] [InSPIRE].

[5] S.C. Park, Minimal gauge inflation and the refined Swampland conjecture, JCAP 01 (2019) 053 [arXiv: 1810.11279] [INSPIRE].

[6] D.Y. Cheong, S.M. Lee and S.C. Park, Higgs Inflation and the Refined dS Conjecture, Phys. Lett. B 789 (2019) 336 [arXiv:1811.03622] [INSPIRE].

[7] M.-S. Seo, de Sitter swampland bound in the Dirac-Born-Infeld inflation model, Phys. Rev. D 99 (2019) 106004 [arXiv: 1812.07670] [INSPIRE].

[8] G. Obied, H. Ooguri, L. Spodyneiko and C. Vafa, de Sitter Space and the Swampland, arXiv: 1806.08362 [INSPIRE].

[9] D. Andriot and C. Roupec, Further refining the de Sitter swampland conjecture, Fortsch. Phys. 67 (2019) 1800105 [arXiv: 1811.08889] [INSPIRE].

[10] S.K. Garg and C. Krishnan, Bounds on Slow Roll and the de Sitter Swampland, JHEP 11 (2019) 075 [arXiv:1807.05193] [INSPIRE].

[11] H. Ooguri, E. Palti, G. Shiu and C. Vafa, Distance and de Sitter Conjectures on the Swampland, Phys. Lett. B 788 (2019) 180 [arXiv:1810.05506] [INSPIRE].

[12] M.-S. Seo, The entropic quasi-de Sitter instability time from the distance conjecture, Phys. Lett. B 807 (2020) 135580 [arXiv:1911.06441] [INSPIRE].

[13] R.-G. Cai and S.-J. Wang, A refined trans-Planckian censorship conjecture, Sci. China Phys. Mech. Astron. 64 (2021) 210011 [arXiv:1912.00607] [INSPIRE]. 
[14] A.G. Cohen, D.B. Kaplan and A.E. Nelson, Effective field theory, black holes, and the cosmological constant, Phys. Rev. Lett. 82 (1999) 4971 [hep-th/9803132] [INSPIRE].

[15] M.-S. Seo, Implication of the swampland distance conjecture on the Cohen-Kaplan-Nelson bound in de Sitter space, arXiv:2106.00138 [INSPIRE].

[16] R. Penrose, Gravitational collapse: The role of general relativity, Riv. Nuovo Cim. 1 (1969) 252 [INSPIRE].

[17] N. Arkani-Hamed, L. Motl, A. Nicolis and C. Vafa, The String landscape, black holes and gravity as the weakest force, JHEP 06 (2007) 060 [hep-th/0601001] [INSPIRE].

[18] M. Montero, T. Van Riet and G. Venken, Festina Lente: EFT Constraints from Charged Black Hole Evaporation in de Sitter, JHEP 01 (2020) 039 [arXiv:1910.01648] [INSPIRE].

[19] M. Montero, C. Vafa, T. Van Riet and G. Venken, The FL bound and its phenomenological implications, JHEP 10 (2021) 009 [arXiv:2106.07650] [INSPIRE].

[20] M. Sher, Electroweak Higgs Potentials and Vacuum Stability, Phys. Rept. 179 (1989) 273 [INSPIRE].

[21] G. Degrassi et al., Higgs mass and vacuum stability in the Standard Model at NNLO, JHEP 08 (2012) 098 [arXiv: 1205.6497] [INSPIRE].

[22] A. Hook, J. Kearney, B. Shakya and K.M. Zurek, Probable or Improbable Universe? Correlating Electroweak Vacuum Instability with the Scale of Inflation, JHEP 01 (2015) 061 [arXiv: 1404.5953] [INSPIRE].

[23] T. Markkanen, A. Rajantie and S. Stopyra, Cosmological Aspects of Higgs Vacuum Metastability, Front. Astron. Space Sci. 5 (2018) 40 [arXiv:1809.06923] [INSPIRE].

[24] J.R. Espinosa et al., The cosmological Higgstory of the vacuum instability, JHEP 09 (2015) 174 [arXiv: 1505. 04825] [INSPIRE].

[25] A.H. Guth, The Inflationary Universe: A Possible Solution to the Horizon and Flatness Problems, Phys. Rev. D 23 (1981) 347 [inSPIRE].

[26] A.D. Linde, A New Inflationary Universe Scenario: A Possible Solution of the Horizon, Flatness, Homogeneity, Isotropy and Primordial Monopole Problems, Phys. Lett. B 108 (1982) 389 [INSPIRE].

[27] A. Albrecht and P.J. Steinhardt, Cosmology for Grand Unified Theories with Radiatively Induced Symmetry Breaking, Phys. Rev. Lett. 48 (1982) 1220 [InSPIRE].

[28] V.F. Mukhanov and G.V. Chibisov, Quantum Fluctuations and a Nonsingular Universe, JETP Lett. 33 (1981) 532 [INSPIRE].

[29] V.F. Mukhanov, H.A. Feldman and R.H. Brandenberger, Theory of cosmological perturbations. Part 1. Classical perturbations. Part 2. Quantum theory of perturbations. Part 3. Extensions, Phys. Rept. 215 (1992) 203 [INSPIRE].

[30] A. Hook, J. Huang and D. Racco, Searches for other vacua. Part II. A new Higgstory at the cosmological collider, JHEP 01 (2020) 105 [arXiv: 1907.10624] [INSPIRE].

[31] Y. Hamada, H. Kawai, K.-y. Oda and S.C. Park, Higgs Inflation is Still Alive after the Results from BICEP2, Phys. Rev. Lett. 112 (2014) 241301 [arXiv:1403. 5043] [INSPIRE].

[32] Y. Hamada, H. Kawai, K.-y. Oda and S.C. Park, Higgs inflation from Standard Model criticality, Phys. Rev. D 91 (2015) 053008 [arXiv:1408.4864] [InSPIRE]. 
[33] Planck collaboration, Planck 2018 results. X. Constraints on inflation, Astron. Astrophys. 641 (2020) A10 [arXiv: 1807.06211] [INSPIRE].

[34] H. Kawai and K. Kawana, Multi-critical point principle as the origin of classical conformality and its generalizations, arXiv:2107.10720 [INSPIRE].

[35] Particle Data Group collaboration, Review of Particle Physics, PTEP 2020 (2020) 083C01 [INSPIRE].

[36] BICEP and KeCK collaborations, Improved Constraints on Primordial Gravitational Waves using Planck, WMAP, and BICEP/Keck Observations through the 2018 Observing Season, Phys. Rev. Lett. 127 (2021) 151301 [arXiv:2110.00483] [INSPIRE].

[37] H.P. Nilles, Supersymmetry, Supergravity and Particle Physics, Phys. Rept. 110 (1984) 1 [INSPIRE].

[38] G.F. Giudice and R. Rattazzi, Theories with gauge mediated supersymmetry breaking, Phys. Rept. 322 (1999) 419 [hep-ph/9801271] [INSPIRE].

[39] CMB-S4 collaboration, CMB-S4 Science Book, First Edition, arXiv:1610.02743 [INSPIRE].

[40] CMB-S4 collaboration, CMB-S4: Forecasting Constraints on Primordial Gravitational Waves, arXiv:2008.12619 [INSPIRE].

[41] LITEBIRD collaboration, LiteBIRD: JAXA's new strategic L-class mission for all-sky surveys of cosmic microwave background polarization, Proc. SPIE Int. Soc. Opt. Eng. 11443 (2020) 114432F [arXiv: 2101.12449] [INSPIRE].

[42] S.H. Henry Tye, D. Wohns and Y. Zhang, Coleman-de Luccia Tunneling and the Gibbons-Hawking Temperature, Int. J. Mod. Phys. A 25 (2010) 1019 [arXiv:0811.3753] [INSPIRE].

[43] S. Coleman, Aspects of Symmetry: Selected Erice Lectures, Cambridge University Press, Cambridge U.K. (1985) [DOI] [INSPIRE].

[44] A.R. Brown and E.J. Weinberg, Thermal derivation of the Coleman-De Luccia tunneling prescription, Phys. Rev. D 76 (2007) 064003 [arXiv:0706.1573] [INSPIRE].

[45] V. Balek and M. Demetrian, Euclidean action for vacuum decay in a de Sitter universe, Phys. Rev. D 71 (2005) 023512 [gr-qc/0409001] [INSPIRE]. 